\title{
COMBS: A Biosurveillance Ecosystem (BSVE) Prototype
}

\author{
Michael E. Cleary*, Erik T. Antelman, Natalya Markuzon, Sarah M. Miller, Timothy A. \\ Postlethwaite and Zahar Prasov
}

The Charles Stark Draper Laboratory, Cambridge, MA, USA

\section{Objective}

The goal of DTRA's Biosurveillance Ecosystem (BSVE) program is to significantly reduce the time required to identify threats to human health and respond appropriately. The Draper Team is developing the Collaborative Overarching Multi-feed Biosurveillance System (COMBS) for BSVE to revolutionize biosurveillance (BSV) capabilities. Analysts will benefit from rapid and thorough information access, as will local public health authorities and individual citizens.

\section{Introduction}

COMBS is facilitating analyst workflows and collaboration, greatly accelerating the management of a bio-event, effectively implementing new capabilities and technologies, and providing opportunities for a wide variety of organizations to contribute data and tools that support their own goals while supporting and governing the ecosystem collaboratively.

\section{Methods}

COMBS provides three key contributions (Figure 1). First, COMBS will revolutionize the analyst's job by providing access to a dynamic and diverse set of heterogeneous BSV data, and tools and apps for understanding, visualizing, and reporting on the implications of the data. Second, the COMBS data sources cloud and app store will enable commercialization and expansion of BSV activities to other stakeholders - Government agencies, DoD, commercial data and tools providers, online advertisers, and the public. Finally, COMBS provides a mechanism for self-sustainment through opportunities for commercialization of data sources, analytic and visualization tools, and online advertising.

Providing a compelling marketplace in information and services that supports participants in achieving their own goals will ensure COMBS services are available when needed. Participation models include that companies will use the informational and computational capabilities to automate and improve many of their own functions. Companies will find a ready market via the App Store. Developing world participants will be able to provide data where little infrastructure currently exists. Innovative developers will have access to a community looking for applications that assist the search for useful information.

\section{Results}

Although the BSVE program is only $25 \%$ complete, the COMBS system already fulfills elements of core user capabilities: Data ingest, Search, Verification, Collaboration \& Communication, Analysis \& Tools, and Reporting. Significant improvement in each of these areas is planned.

The prototype cloud-based COMBS dashboard (Figure 2) is where an analyst will create their desired working environment by selecting desired apps from the app store. The dashboard is fully customizable, enabling a user to drag and drop a variety of apps that will enable key capabilities.

\section{Conclusions}

The Draper team is developing COMBS to facilitate earlier detection of and more effective response to biological events by providing:
A flexible cloud-based data integration platform supporting collection and integration of biosurveillance information from a variety of Governmental and other sources (including social media and news reports),

- $\quad$ A user-customizable, easy-to-navigate analyst workbench,

- Collaboration tools supporting joint analysis, and preparation of reports and briefings, and

- $\quad$ A BSVE self-sustainment mechanism with commercialization of data sources, analytic and visualization tools, and advertising.

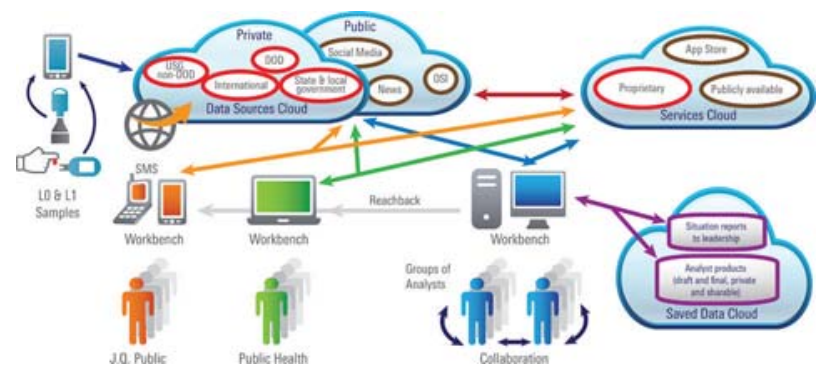

Figure 1: COMBS Operational View

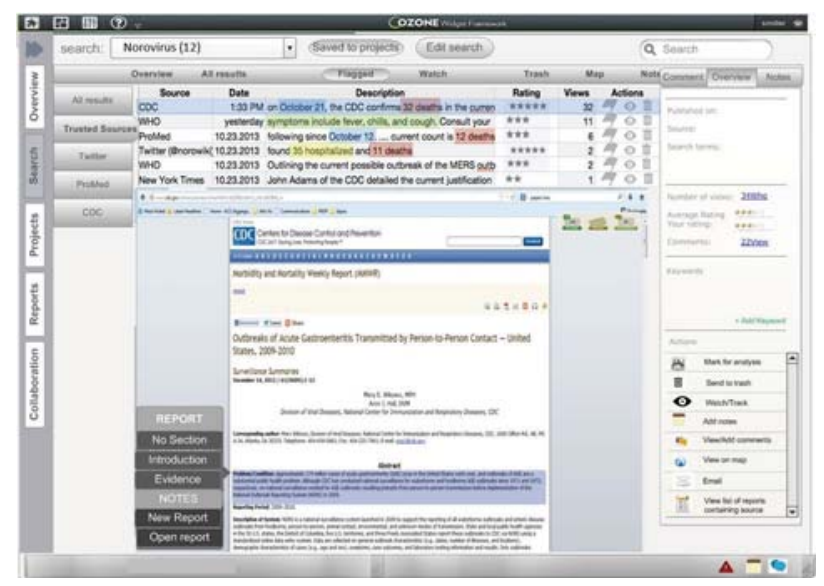

Figure 2: COMBS Prototype Dashboard Concept

\section{Keywords}

biosurveillance; public health; identify threats; analyst workbench

\section{Acknowledgments}

This work is sponsored by the Defense Threat Reduction Agency (DTRA), through contract N66001-13-C-2025, established and administered by the Space and Naval Warfare Systems Center Pacific.

Copyright (C) 2013 by The Charles Stark Draper Laboratory, Inc. All copies or adaptations of this work must include this notice in its entirety, and all changes made to the original Work must be clearly labeled or otherwise identified.

\section{*Michael E. Cleary}

E-mail: mcleary@draper.com 